\title{
Convergence and oscillation in standardization games
}

\author{
Emmanuelle Auriol* and Michel Benaim**
}

\begin{abstract}
Auriol and Benaim (2000) studied in a model inspired by evolutionary game theory, how standards and norms emerge in decentralized economies when there are two standards. They showed that the decentralized adoption process always converges toward a stable equilibrium (possibly an incompatibility one). This paper explores the robustness of Auriol and Benaim (2000) convergence results. It shows that with more than two standards the decentralized adoption process does not necessarily converge. It can oscillate and describe cycles.
\end{abstract}

Classification Codes: C73, D62, L1.

\section{Introduction}

Modern economies operate under the principle of labor division and task specialization. It allows individuals and firms to take advantage of increasing returns to scale in work but on the other hand raises serious coordination issues. It requires a high level of standardization to make specialized entities fit together. The inability of people to coordinate themselves and to elect a common standard creates high collective and individual costs ${ }^{1}$. This paper studies how decentralized individuals choose a standard. The issue is coordination because the individual benefit of adoption depends on whether other agents choose the same standard. This is for instance the case for abstract communication standards such as international languages, traffic rules, mathematical or writing symbols. This is also the case for physical standards such as electric norm, computer operating systems, telephone or facsimile equipment. In a different way it applies for commodities that require the consumption of complementary goods such as personal computers, VCR's, stereo components or video games (because the availability of complementary goods depends on the number of users of primary goods). Finally as human is social, needs for standardization arise for social reasons.

* ARQADE and IDEI, University of Toulouse I, Ad: IDEI, place Anatole-France, 31042 Toulouse Cedex, France. E-mail: eauriol@cict.fr

** Department of Mathematics, University of Cergy-Pontoise, 2 av. A. Chauvin, 95302 Cergy-Pontoise Cedex.

Keywords: Network externalities, market structure, dynamics.

${ }^{1}$ For instance the costs linked to the development of gateway technologies or "converters", are usually high, more importantly their performances are low. 
Choosing a standard is a collective problem. How we operate these collective choices is not a trivial issue. In a decentralized economy the agents need a communication system to learn about the economic decisions of others and coordinate her decision with theirs. Usually prices play this role. Then the classical economic literature focuses on the problem of coordination among anonymous agents through markets and prices. However in the case of non-proprietary technologies with network externalities the relevant coordinating mechanism is not the price. The individuals take their decisions according to the market shares of each technology. In this case the non-cooperative game theory literature relies on the Nash equilibrium concept to predict coordination - or anti-coordination among the strategic agents. These two approaches are static in essence (they rely on fixed point theorems). Though relevant in many situations and extremely convenient in use, their static nature is an obstacle to study important aspects of the problem at hand.

Indeed in decentralized economies standardization is in general the result of individual choices despite the collective - social - nature of the decision. This yields a problem because society lasts longer than individuals. That is, there are two time scales: a short one for individuals and a longer one for society. The coexistence of these two time scales affects economic outcome. In particular, since individuals are transient and aware of it, they are present minded. It implies that from the long run - social - perspective they behave myopically. Endowed with a short life they deal the best they can with the present, taking the past as given. Their present behavior however influences the future. Each adopter being concerned with her present economic situation, she does not internalize the cost she imposes (or the benefit she brings) on future consumers while (not) adopting a given standard. We may wonder whether successive myopic adoptions (they might be rational from individuals short run point of view, but not from the social long run one) lead to some kind of unconscious maximizing collective behavior? In other words is there any long living invisible hand at work?

The industrial organization literature on technological choices in the presence of positive coordination externalities, then referred to as network externalities, does not address this question ${ }^{2}$. It belongs to the classic - static - game theoretic approach. It considers the problem of equilibrium existence (i.e., characterization) but not the convergence issue. This problem is not trivial however. A recurrent theme in the classic network externalities literature is that increasing returns of adoption generates a multiplicity of equilibria (at least as much as available technologies) and inefficiencies (i.e., a suboptimal level of compatibility or standardization on the wrong technology). In most of the cases the authors are able to find these equilibria analytically but since their models are static, they fail to solve what Arthur (1988) calls the selection problem. That is, how a particular equilibrium comes to prevail. Arthur $(1988,1989)$ proposes to answer this question by studying the adoption process of competing technologies subject to network externalities in stochastic models. Arthur's idea is that increasing returns tend to magnify "historical events" (path-dependency property) and to trap the dynamics into dominated standard (lock-in property). He illustrates these ideas in a random walk model with absorbing barriers. In such a model standardization occurs with probability one (lock-in)

\footnotetext{
${ }^{2}$ See for instance Farrell and Saloner (1985) and Katz and Shapiro (1985).
} 
but which technology is going to prevail is not predictable (path-dependency). Since the adoption process is non-ergodic and self-reinforcing, Arthur's contribution suggests that standardization in decentralized economy is totally unpredictable. This contradicts the intuitive perception that market driven standardization is not that unpredictable, nor inefficient. From a theoretical point of view it remains to explain how a particular equilibrium prevails; how a multitude of independent, all negligible individuals actually coordinate.

This question lies at the core of the growing literature on evolutionary game theory and social learning. This literature, that aims to explain how equilibrium emerges in games that have multiple Nash equilibria, focuses on convergence results. Among the founding papers of this literature there are Fudenberg and Kreps (1993), Ellison (1993), Kandori et al. (1993), and Crawford (1995) to name just a few. For surveys of the literature see the books by Weibull (1995) and Fudenberg and Levine (1997). Following this original and fruitful line of research, the present paper studies within a dynamical model how equilibrium is reached in a standardization game with multiple standards. It focuses on the coordination problem faced by negligible and anonymous individuals who have to choose among several non-proprietary standards. The agents perceive themselves as being negligible with respect to market shares. The dynamic dimension is captured by the fact that they adopt sequentially and in a random order. The two time scales - individual/social - is captured by the fact that "new born" adopters who inherit a state of standardization from their predecessors are not forward looking. Endowed with a short life, they optimize in the short run taking the past as given. In other words adopters are myopic.

The coordination problem we consider admits in its static version multiple equilibria (in general as many as the number of available standards - everybody coordinates on either one of the standards - and sometimes incompatibility ones). When there are only two standards in competition, introducing the dynamics helps to solve the selection problem: only stable equilibrium emerges in the long run. This result has been established by Auriol and Benaim (2000) for utility functions that depend only on the market share of the adopted technology. The paper generalizes this convergence result to general utility functions. We next analyze the emergence of standards in the long run when there are more than two standards in competition. We show that with three standards the standardization process might oscillate between equilibria and never converge. In other words, the convergence result is not robust to the introduction of several standards.

This paper is organized as follows. Part 2 presents the model and the main assumptions. Part 3 presents the dynamics of the model. Section 3.1 focuses on the two standards case. It presents general results about the convergence of the underlying stochastic process. Section 3.2 focuses on the three standards case. It presents an example with oscillations. Part 4 summarizes our results and offers some concluding remarks.

\section{The model}

The notation and the model are based on Auriol and Benaim (2000). We consider a model in which there are $n \geq 2$ incompatible standards from which to choose, $S_{1}$, 
$S_{2}, \ldots S_{n}$. The standards are identified by an index $i=1, \ldots, n$. The adopters have an inelastic demand for one unit of the commodity. They have heterogeneous preferences over alternatives $i=1, \ldots, n$. More precisely we assume that they are identified by their location $\delta$ in the $n-1$ dimensional unit simplex with the standards exogenously located at the extreme points. Let

$$
\Delta^{n-1}=\left\{x=\left(x_{1}, x_{2}, \ldots x_{n}\right) \in \mathbf{R}^{n}: x_{i} \geq 0, \sum_{i=1} x_{i}=1\right\}
$$

denote the $n-1$ dimensional unit simplex. Each adopter is parameterized by $\delta \in \Delta^{n-1}$ which can be interpreted as a taste parameter which indicates in the continuum of possible good characteristics the one preferred by the agent. It is the "ideal" standard of the adopter. Each adopter has a preference for one of the standards (the closer he is to a technology the more he likes it). But despite this location predilection he may choose another technology in order to benefit from the network externalities. Let $x \in \Delta^{n-1}$ denoted a market share profile of the standards where $x_{i}$ denotes the market share associated to standard $S_{i}$. The preferences of a type $\delta$ agent can be represented by the following utility functions

$$
x \in \Delta^{n-1} \rightarrow \delta_{i} U_{i}(x)
$$

where $U_{i}$ is a positive function representing the net utility function associated with standard $S_{i}$. This paper is not considering the issue of strategic pricing by sellers. This assumption is consistent with either the standards being non proprietary, or the promoters of the standard being engaged in Bertrand competition. However the analysis remains valid even if the prices are not set at marginal cost as long as the net utility functions satisfy the assumptions of the paper.

Term $\delta_{i}$ can be interpreted as a discount factor which reflects the adaptation cost incurred by the agent when she cannot consume her ideal standard, located at $\delta$, but an imperfect substitute. Note that the utility derived from the adoption varies across technologies (in general, $U_{i}(x) \neq U_{j}(x)$ for $i \neq j$ ). We assume that the utility functions entail positive network externalities. That is, the utility associated with the adoption of technology $i$ is positive and strictly increasing with the market share $x_{i}$ (the essence of network externalities):

$$
\frac{\partial U_{i}(x)}{\partial x_{i}}>0 \quad \forall x_{i} \geq 0 \quad(i=1, \ldots, n) .
$$

Proposition 1. An agent located at $\delta=\left(\delta_{1}, \ldots, \delta_{n}\right)$ confronted with the vector of market share $x=\left(x_{1}, \ldots, x_{n}\right)$ will adopt standard $S_{i}$ if and only if:

$$
\delta_{i} U_{i}(x)=\max _{j} \delta_{j} U_{j}(x)
$$

Proof. See Appendix 1.

This simple framework captures the basic trade-off between diversity and compatibility. So, once an agent enters the market, she compares the current market shares of the 
different standards and, based on that, she makes her choice. Conditionally to the past she adopts $S_{i}$ if $\delta_{i} U_{i}(x)=\max _{j} \delta_{j} U_{j}(x)$. Such behavior is referred to as myopic because the agent does not try to integrate in her decision the future evolution of the standard. That is, as in Auriol and Benaim (2000), we make the extreme assumption that the agents' discount rate is infinite. In the case of standard adoption this might be explained by the fact that they are constantly confronted with innovations. Anticipating obsolescence they discount the future heavily. This is especially true when the obsolescence or break down rate is perceived to be much higher than market shares' motion. In practice experiments suggest that individuals display hyperbolic discounting function ${ }^{4}$. As a result of the infinite discount rate, once they enter the market individuals' adoption choice is deterministic. On the contrary, the law driving their entry, and thus the adopter sequence, is assumed to be exogenous and random. It can be explained by the need to replace obsolete (broken) technology or by a demographic law. To keep the analysis simple we assume that each agent is equally likely to enter the market at time $t$. That is the candidates for adoption are uniformly distributed over $\Delta^{n-1}$.

$$
\delta \text { is uniformely distributed over } \Delta^{n-1} .
$$

In the next section, we present the dynamics of the market shares allocation.

\section{The dynamics}

The dynamics of the model are as follows. At each round of adoption, identified by $t$, $t+1$, and so on, an agent, identified by $\delta$, is drawn randomly from the population of potential adopters. This agent decides, according to the current market shares and his individual preference, which technology to choose. The sequence of adopter is exogenous and random, but the adoption choice is endogenous and, for a given consumer at a given date, deterministic (at date $t+1$ consumer $\delta$ adopts $S_{i}$ if $\delta_{i} U_{i}(x)=\max _{j} \delta_{j} U_{j}(x)$ ). Remark that in this model the rate at which adopters arrive on the market, contrary to the order that may matter, is unimportant. The relevant time is not that of the clock. Period $t$ designates the $t$ th round of adoption. The probability that technology $i$ is going to be chosen at round $t+1$ of adoption is derived in the next proposition. For $i \neq j$ define the function

$$
\alpha_{j}^{i}(x)=\frac{U_{i}(x)}{U_{j}(x)} .
$$

Proposition 2. Suppose $\delta$ is a random variable uniformly distributed over $\Delta^{n-1}$. Let $x \in \Delta^{n-1}$ denote the vector of market share. Then

$$
\begin{gathered}
P_{i}=\operatorname{Pr}\left(\text { type } \delta \text { agent chooses } S_{i}\right)=\operatorname{Pr}\left[\delta_{i} U_{i}(x)=\max _{j} \delta_{j} U_{j}(x)\right] \\
=1+\sum_{k=1}^{n-1}\left[(-1)^{k} \sum_{j_{1}<j_{2}<\ldots<j_{k} \in J^{i}} \frac{1}{\alpha_{j_{1}}^{i}(x)+\ldots+\alpha_{j_{k}}^{i}(x)+1}\right]
\end{gathered}
$$

\footnotetext{
${ }^{4}$ For a review of the literature and of the empirical evidences on this point see Ainslie (1992) and Loewenstein and Prelec (1992).
} 
where

$$
J^{i}=\{1, \ldots, n\} \backslash\{i\}
$$

Proof. See Appendix 2.

In what follows we are going to focus on two cases, $n=2$ and $n=3$.

- For $n=2$ we deduce from Proposition 2

$$
P_{1}=\operatorname{Pr}\left(\text { type } \delta \text { agent chooses } S_{1}\right)=1-\frac{1}{\alpha_{2}^{1}(x)+1}=\frac{U_{1}(x)}{U_{1}(x)+U_{2}(x)} .
$$

This generalized the Auriol and Benaim (2000) model where $U_{i}(x)=U_{i}\left(x_{i}\right)(i=1,2)$ which gives

$$
P_{1}=\frac{U_{1}\left(x_{1}\right)}{U_{1}\left(x_{1}\right)+U_{2}\left(1-x_{1}\right)}
$$

- For $n=3$ we deduce from Proposition 2

$$
P_{1}=\operatorname{Pr}\left(\text { type } \delta \text { agent chooses } S_{1}\right)=1-\frac{1}{\alpha_{2}^{1}(x)+1}-\frac{1}{\alpha_{3}^{1}(x)+1}+\frac{1}{\alpha_{2}^{1}(x)+\alpha_{3}^{1}(x)+1} \text {. }
$$

Let $N_{i}^{0} \geq 1$ be the initial number of adopters of standard $i=1, \ldots, n$. From a technical point of view, this can be any integer larger than one. Moreover, let $N_{i}^{t}$ denote the cumulative number of agents that have adopted standard $i$ at time $t$, and $N^{t}$ denote the cumulative number of adoptions which have occurred till time $t$. Observe that $N^{t}=\sum_{i=1}^{n} N_{i}^{0}+t$. The market share of standard $i$ at time $t$ is $x_{i}^{t}=N_{i}^{t} / N^{t}$.

Let $P_{i}^{t}$ denotes the probability that standard $i$ will be selected at round $t+1$ of adoption (see Prop. 1).

$$
\begin{aligned}
P_{i}^{t} & =\operatorname{Pr}\left(\text { type } \delta \text { agent chooses at round } t+1 \text { of adoption } S_{i}\right) \\
& =\operatorname{Pr}\left(\delta_{i} U_{i}\left(x^{t}\right)=\max _{j} \delta_{j} U_{j}\left(x^{t}\right)\right) .
\end{aligned}
$$

The dynamics of market share are given by the following equation $(i=1, \ldots, n)$ :

$$
x_{i}^{t+1}=\frac{1}{N^{t}+1} \begin{cases}N_{i}^{t}+1 & \text { with probability } P_{i}^{t} \\ N_{i}^{t} & \text { with probability } 1-P_{i}^{t} .\end{cases}
$$

Let $\alpha^{t}=\frac{1}{N^{t}+1}$. Then system (1) can be rewritten in the form:

$$
x_{i}^{t+1}= \begin{cases}\left(1-\alpha^{t}\right) x_{i}^{t}+\alpha^{t} & \text { with probability } P_{i}^{t} \\ \left(1-\alpha^{t}\right) x_{i}^{t} & \text { with probability } 1-P_{i}^{t} .\end{cases}
$$

We derive from system (2) the expected motion of market share with respect to the current state $x_{i}^{t}$. We get:

$$
E\left(x_{i}^{t+1} \mid x_{i}^{t}\right)=x_{i}^{t}+\alpha^{t}\left(P_{i}^{t}-x_{i}^{t}\right)
$$


The interpretation of equation (3) is straightforward. The market share of standard $i$ increases in expectation if the probability of standard $i$ being adopted is greater than its current market share. Otherwise, it declines; term $\alpha^{t}\left(P_{i}^{t}-x_{i}^{t}\right)$ is positive when $P_{i}^{t}>x_{i}^{t}$ and negative when $P_{i}^{t}<x_{i}^{t}$. Indeed, if the market share of one standard is much smaller than its probability of being adopted, then this standard will be chosen on average more than proportionally to its current market share, and thus its market share will grow. By the same reasoning, if the market share of one standard is much larger than its probability of being adopted, then this standard's market share will decline. Intuition suggests that the stochastic system should converge in the long run towards a state where adoption probability and market share equalize. In other words, the long run behavior of the stochastic process (2) is related to the behavior of the following deterministic dynamical system defined on $\Delta^{n-1}$ :

$$
\frac{\mathrm{d} x}{\mathrm{~d} t}=-x+P(x)
$$

where

$$
\begin{gathered}
P: \Delta^{n-1} \rightarrow \Delta^{n-1}, \\
x \rightarrow P(x)=\left(P_{1}(x), \ldots, P_{n}(x)\right)
\end{gathered}
$$

is defined Proposition 2.

Then with the stochastic process (2), we associate the deterministic dynamical system 4 which can be equivalently written (taking into account the condition $x_{1}=1-\sum_{i=2}^{n} x_{i}$ )

$$
\frac{\mathrm{d} x_{i}}{\mathrm{~d} t}=-x_{i}+P_{i}(x), \quad i=2 \ldots n .
$$

In what follows we check on two cases that the long run behavior of the stochastic process (1) is related to the behavior of the deterministic dynamical system (4). This implies that if the deterministic dynamical system (4) always converge towards a stable equilibrium, then the stochastic process (1) will converge. On the other hand, if the deterministic dynamical system (4) admits periodic solutions, then one has to expect a periodic behavior for the stochastic process (1). We next define what a stable equilibrium is. Let

$$
\mathcal{E}=\left\{\left(x \in \Delta^{n-1}\right): P(x)=x\right\}
$$

denote the equilibrium set of (4). An equilibrium $x \in \mathcal{E}$ is called linearly stable if all the eigenvalues of the matrix

$$
D P(x)=\frac{\partial P_{i}(x)}{\partial x_{j_{2 \leq i, j \leq n}}}
$$

have their real parts $<1$. It is called linearly unstable if at least one eigenvalue has a real part $>1$.

In the sequel of the paper we show that with two standards in competition whatever the utility functions the stochastic adoption process always converge. This generalizes the Auriol and Benaim (2000) convergence result. On the other hand, when there are three 
standards in competition we show that this convergence result does not hold anymore. The stochastic adoption process can describe cycles.

Remark. The network externalities literature considers the number of adopters rather than the market shares. This distinction is empty in these models where the population of adopters is fixed. Conditionally on $N$ the total population of adopters, it is equivalent to know the market share of standard $k$ or its number of adopters. Here as we consider a dynamical model where the cumulative number of adoptions $N^{t}$ increases over time, the market share of one standard and its number of users do not convey the same information (it is not the same to know that there are 1 million of users of one standard when this standard represents $70 \%$ of total users and when it represents $10 \%$ only). Since in decentralized economies it is impossible to keep track of the exact number of users of one standard, and since these numbers are too large anyway to be easily advertised or memorized, the information in general available in the economy concerns market shares. As a result individuals base their adoption decisions on these values ${ }^{5}$.

\subsection{Convergence with 2 standards}

The next Proposition establishes that the vector of market shares $x^{t}=\left(x_{1}^{t}, x_{2}^{t}\right)$ of system (2) converges towards the stable equilibria of (4) $\left(x_{1}^{*}=P_{1}\left(x_{1}^{*}, x_{2}^{*}\right), x_{2}^{*}=P_{2}\left(x_{1}^{*}, x_{2}^{*}\right)\right)$.

Proposition 3. i) With probability one

$$
\lim _{t \rightarrow \infty} \operatorname{dist}\left(x^{t}, \boldsymbol{\varepsilon}\right)=0 .
$$

ii) Suppose $\varepsilon$ is finite (or countable). Then $x_{t}$ converges almost surely toward an equilibrium point $x *$.

iii) Let $x *$ denote an equilibrium. If $x *$ is linearly stable, then

$$
P\left(x_{t} \rightarrow x *\right)>0 .
$$

iv) If $x *$ is linearly unstable, then

$$
P\left(x_{t} \rightarrow x *\right)=0 .
$$

\footnotetext{
${ }^{5}$ Even if the market share assumption is consistent with facts, we want to emphasize that in the case $n=2$ considering a fixed population of adopters over time (rather than an increasing one) would lead to the same qualitative results while involving different mathematical analysis. In this new setting the current number of users of one of the two standards is assumed to be fix and large $\left(N^{t} \equiv N \forall t\right)$. The market share of standard $k$ at time $t$, which in this case is equivalent to the number of adopters, is $x_{k}^{t}=N_{k}^{t} / N$. Let $\alpha=1 / N$, by similar arguments as the ones developed to obtain equation (3) we obtain: (3') $E\left(x_{k}^{t+1} \mid x_{k}^{0} \ldots, x_{k}^{t}\right)=$ $x_{k}^{t}+\alpha\left(P_{k}^{t}-x_{k}^{t}\right)$. The main difference is that the process ( $\left.3^{\prime}\right)$ is now an homogeneous Markov process (i.e. time independent) and therefore we cannot expect to obtain almost sure convergence properties as stated in Proposition 3. However the long term behavior of this new process is qualitatively very similar to the long term behavior of the process (3). That is, when $t$ goes to infinity the market share $x_{k}^{t}$ tends to concentrate with a large probability in the neighborhood of the stable equilibria of (4). This result is a consequence of the Large deviations Theory for recursive algorithms (see Dupuis, 1998).
} 
Proof. The proof is a consequence of results on stochastic approximations: The almost sure convergence (assertions $i$ ), ii)) follows from the fact that (5) is a one dimensional equation combined with Corollary 6.7 in Benaim (1999). Statements iii) and iv) are direct consequences of Proposition 7.5 and Theorem 9.1 in Benaim (1999).

Proposition 3 generalized the Auriol and Benaim (2000) convergence result to the case where the utility functions depend on the total vector of market shares. It thus establishes that with two standards in competition the stochastic decentralized process of adoption (1) always converges towards a stable equilibrium of (4). This result olds independently of the particular shape of the utility functions. However to analyze the exact nature of the equilibrium it is necessary to be more specific about the utility functions. To illustrate this point we consider next an example based on Auriol and Benaim (2000).

Example. In Auriol and Benaim (2000) framework, the net utility functions depend on the market share of the relevant standard only. That is:

$$
U_{i}(x)=U_{i}\left(x_{i}\right) \quad i=1,2 .
$$

Moreover it is assumed that

$$
U_{i}(0)=0 \quad \text { and } \quad \frac{\mathrm{d} U_{i}\left(x_{i}\right)}{\mathrm{d} x_{i}}>0 \quad \forall x_{i} \geq 0 \quad i=1,2 .
$$

Assumption A1 captures the "network externalities" assumption. The larger the market share of a given standard, the greater the gross benefit of adopting it. On the other hand, if there is no adopter of one standard it has no use, the gross utility is null. Under assumption A1 and A2 Auriol and Benaim (2000) show that standardization outcome depend on the adopters' attitude to problems raised by incompatibility which is related to the concavity/convexity of the utility functions. To illustrate their results let consider that $U_{1}\left(x_{1}\right)=U\left(x_{1}\right)$, and $U_{2}\left(x_{2}\right)=\beta U\left(x_{2}\right)$, with $\beta>0$, and $U($.$) either convex or$ concave. This formalization allows us to parameterize the problem. That is, we can study in function of the parameter $\beta$ the different standardization equilibria which might emerge in the long run. Note that for $\beta \leq 1$, standard 1 perfoms better than standard 2 , and that it is the contrary for $\beta \geq 1$.

Consider first the convex/linear case (i.e., $U($.$) convex or linear in x$ ). It is easy to check that the conditions $A N 1, A N 2$, and $A N 3$ in Auriol and Benaim (2000) become ${ }^{6}$ :

$$
\begin{array}{ccc}
A N 2 & A N 1 & A N 3 \\
\beta \leq \frac{U^{\prime}(0)}{U(1)} & \frac{U^{\prime}(0)}{U(1)}<\beta<\frac{U(1)}{U^{\prime}(0)} & \frac{U(1)}{U^{\prime}(0)} \leq \beta .
\end{array}
$$

By virtue of Proposition 3, and the above definition of stability, standardization occurs on standard 1 under $A N 2$, on standard 2 under $A N 3$, and either on standard 1 or 2 (path dependency) under $A N 1$. The interpretation of this result is as follows. When the utility functions are convex in market share, individuals display aversion to incompatibility. When standardization failure puts individuals at risk, they display intolerance to incompatibility. This covers standards that raise safety concern such as electrical norms, drug

\footnotetext{
${ }^{6}$ The convexity of $U(x)$ implies $U(1) \geq U^{\prime}(0)$.
} 
formula, air, see or road traffic rules and signs. In this case standardization is systematic, but it is not always possible to predict which standard will prevail. In their haste to coordinate, they sometimes erroneously select the dominated standard.

When the utility functions are linear, individuals display neutrality to incompatibility. Neutrality to incompatibility arise when the benefit received from the adoption of a particular technology or standard increases proportionally with the aggregate number of agents who adopt the same standard. The consumers search for compatibility because it gives them access to a larger physical network. For instance, the value of access to a facsimile equipment, real estate listing service, telephone network, yellow page directory, or electronic mail service, depends on the total number of other households or businesses with similar access. In this case standardization occurs and is always predictable. Individuals always select the best standard. There is neither path-dependency, nor a lock-in problem. In fact the standardization outcome is optimal.

Let next consider the concave case (i.e., $U($.$) strictly concave in x$ ). It is easy to check that the conditions $T 1, T 2$, and $T 3$ in Auriol and Benaim (2000) become ${ }^{7}$ :

$T 2$

$$
\beta \leq \frac{U(1)}{U^{\prime}(0)}
$$

$T 1$

$$
\frac{U(1)}{U^{\prime}(0)}<\beta<\frac{U^{\prime}(0)}{U(1)}
$$

\section{$T 3$}

$$
\frac{U^{\prime}(0)}{U(1)} \leq \beta \text {. }
$$

By virtue of Proposition 3 and the definition of stability, the standardization equilibrium is always predictable. Standardization occurs on standard 1 under $T 2$, on standard 2 under $T 3$, and there is an incompatibility equilibrium under $T 1$. That is, when individuals display tolerance to incompatibility, which corresponds to concave utility functions, the adoption process is predictable. The basic trade off between diversity and network externalities does not necessarily lead to standardization though. Nevertheless, since in this case consumers take time to experiment they never take a bad for a good. When standardization occurs it is always on the dominant standard. Tolerance to incompatibility occurs when the standardization benefits are indirect. When the consumption of a durable good, "hardware", requires the consumption of complementary goods, "software", the utility that an agent derives from the acquisition of the durable good is enhanced as the availability of complementary goods increases. An agent is concerned with the number of other agents purchasing similar durable good because a larger inplace base of users of a particular hardware implies a greater variety of compatible software. This concerns personal computers, video cassette players-recorders, stereo components, video games.

The convergence results, which depend upon the value of $\beta$, are summarized Figure 1. The figure contrasts the different standardization outcomes which are going to emerge in the long run in function of $\beta$ when the utility functions are concave and when they are convex. The dash lines represent the unstable equilibria, the continuous lines the stable equilibria.

\footnotetext{
${ }^{7}$ The concavity of $U(x)$ implies $U^{\prime}(0) \geq U(1)$.
} 

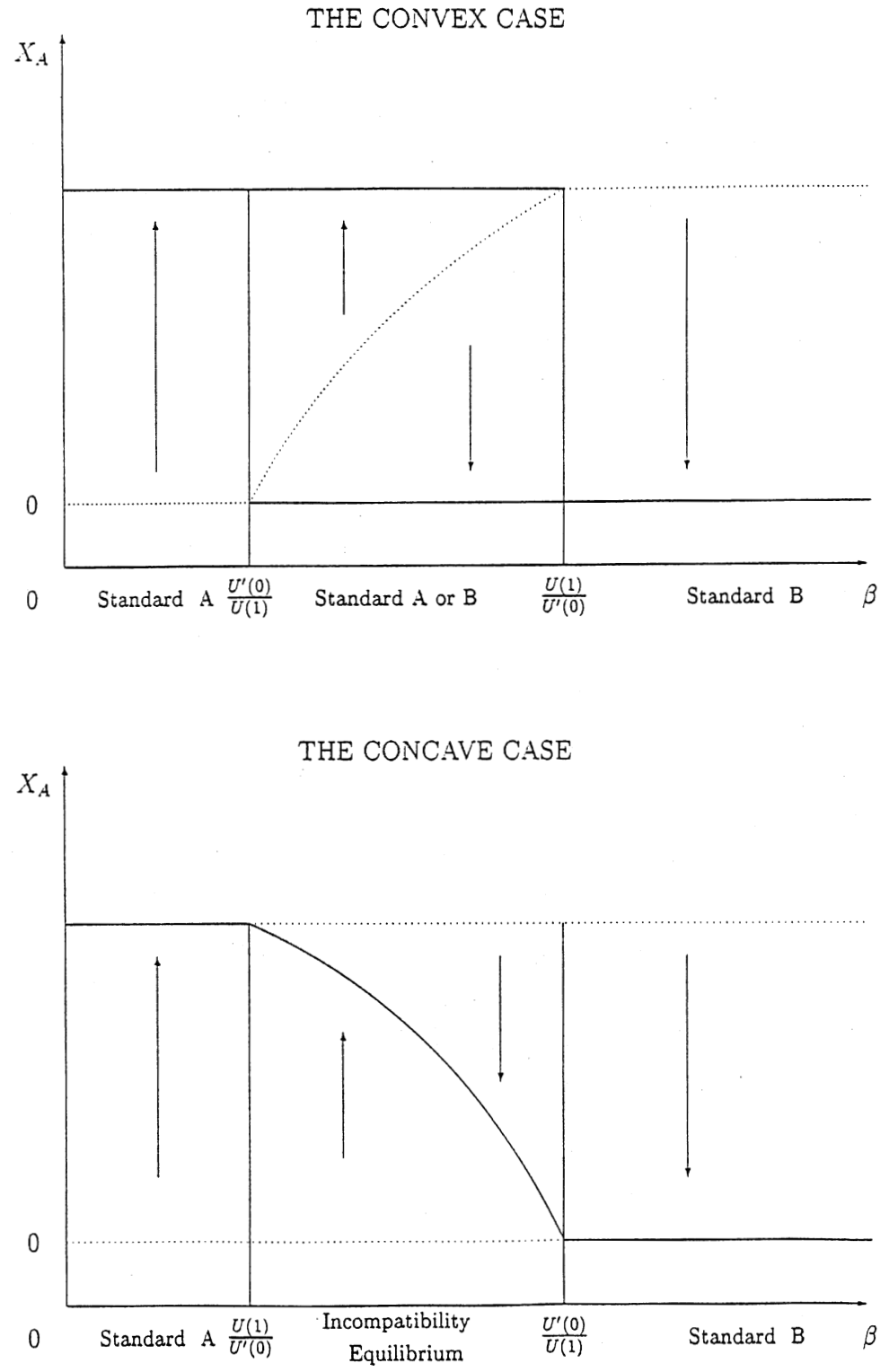

Fig. 1.

We now turn to the case $n=3$.

\subsection{Oscillations with 3 standards}

The main purpose of this section is to show that, in contrary to the global convergence result given by Proposition 3, the dynamics associated to a system with 3 standards can 
oscillates with positive probability. In order to prove this result it is sufficient to find an example where indeed oscillations occur.

To analyze the 3 standards system it is convenient to introduce the following variables

$$
x=x_{2} \quad \text { and } \quad y=x_{3},
$$

so that

$$
x_{1}=1-x-y \text {. }
$$

We then define the functions

$$
\alpha=\alpha(x, y)=\alpha_{2}^{1}, \quad \text { and } \quad \beta=\beta(x, y)=\alpha_{3}^{1} .
$$

It is then easy to verify that $\alpha_{1}^{2}=1 / \alpha, \alpha_{3}^{2}=\beta / \alpha, \alpha_{1}^{3}=1 / \beta$ and $\alpha_{2}^{3}=\alpha / \beta$. Therefore the dynamical system (5) takes the form

$$
\begin{aligned}
& \frac{\mathrm{d} x}{\mathrm{~d} t}=-x+p(\alpha(x, y), \beta(x, y)) \\
& \frac{\mathrm{d} y}{\mathrm{~d} t}=-y+p(\beta(x, y), \alpha(x, y))
\end{aligned}
$$

where

$$
p(\alpha, \beta)=1-\alpha\left(\frac{1}{\alpha+1}+\frac{1}{\alpha+\beta}-\frac{1}{\alpha+\beta+1}\right) .
$$

A full analysis of this dynamics is hopeless, nevertheless a local analysis is possible.

Let $m$ denote the points with coordinates $x=y=1 / 3$. From now on we will assume that the function $\alpha, \beta$ satisfy the property that $\alpha(m)=\beta(m)=1$. In other words: the net utility functions of the standards are identical when the market shares are identical. Under this assumption it is not hard to verify the following preliminary result.

Lemma 1. The Jacobian matrix of the vector field

$$
(x, y) \rightarrow(-x+p(\alpha, \beta),-y+p(\beta, \alpha))
$$

at point $m$ is given by

$$
\left(\begin{array}{cc}
-1+\frac{5}{36}\left(-2 \alpha_{x}+\beta_{x}\right) & -\frac{5}{18}\left(-2 \alpha_{y}+\beta_{y}\right) \\
-\frac{5}{18}\left(\alpha_{x}-2 \beta_{x}\right) & -1-\frac{5}{18}\left(\alpha_{y}-2 \beta_{y}\right)
\end{array}\right)
$$

where $\alpha_{x}$ (respectively $\alpha_{y}, \beta_{x}, \beta_{y}$ ) stands for $\partial_{x} \alpha(m)=\partial \alpha(m) /(\partial x)$ (respectively $\partial_{y} \alpha(m), \partial_{x} \beta(m), \partial_{y} \beta(m)$.

Proof. By the chain rule $\partial_{x} f(\alpha, \beta)=\left(\partial_{\alpha} f\right) \alpha_{x}+\left(\partial_{\beta} f\right) \beta_{x}$. At point $(1,1) \partial_{\alpha} f(1,1)=$ $-5 / 18$ and $\partial_{\beta} f(1,1)=5 / 36$. Thus $\partial_{x} f(\alpha, \beta)=5 / 36\left(-2 \alpha_{x}+\beta_{x}\right)$. The other computations are similar.

Let $T$ and $D$ denote respectively the trace and determinant of the matrix given by Lemma 1. That is

$$
T=-2+\frac{5}{36}\left(\beta_{x}-2 \alpha_{x}+\alpha_{y}-2 \beta_{y}\right)
$$


and

$$
D=\left(-1+\frac{5}{36}\left(\beta_{x}-2 \alpha_{x}\right)\right)\left(-1+\frac{5}{36}\left(\alpha_{y}-2 \beta_{y}\right)\right)-\left(\frac{5}{36}\right)^{2}\left(\beta_{y}-2 \alpha_{y}\right)\left(\alpha_{x}-2 \beta_{x}\right) .
$$

We have the following local result:

Proposition 4. Suppose $D \neq 0$. If $T<0$ and $D>0$ then $x_{t} \rightarrow m$ with positive probability, while in the other situations $x_{t}$ never converges to $m$.

Proof. If $D \neq 0$, the condition $T<0$ and $D>0$ is equivalent to the linear stability of $m$. The result then follows from Propositions 7.5 and Theorem 9.1 in Benaim (1999).

As an illustration of Proposition 4 suppose that the three standards have the same net utility function. That is $U_{i}(x)=V\left(x_{i}\right)$. Let $t=-V^{\prime}(1 / 3) / V(1 / 3)$. Then

$$
T=-2\left(1+\frac{5}{12} t\right) \quad \text { and } \quad D=\left(1+\frac{5}{12} t\right)^{2} .
$$

In view of Proposition 4 we then have

$$
x_{t} \rightarrow m
$$
with positive probability provided $V^{\prime}\left(\frac{1}{3}\right)<\frac{12}{5} V\left(\frac{1}{3}\right)$ while, if $V^{\prime}\left(\frac{1}{3}\right)>\frac{12}{5} V\left(\frac{1}{3}\right), x_{t}$ never
converges to $m$.

Proposition 4 establishes that if $m=(1 / 3,1 / 3,1 / 3)$ is a stable equilibrium of the deterministic dynamical system given by (6), then the stochastic dynamical system 1 converges with positive probability to $m$. More generally the long run behavior of the stochastic dynamical system 1 can be related to the behavior of the deterministic dynamical system 6 . This implies that when the deterministic dynamical system always converges to an equilibrium, the stochastic system also converge. By the same reasoning if the deterministic dynamical system describes cycles, the long run behavior of the stochastic system is likely to be cyclical. This result is proven in the next section.

\subsubsection{Oscillation with 3 standards}

In order to shows the non convergence result in the case of 3 standards we suppose that the function $\alpha$ and $\beta$ have the form

$$
\alpha(x, y)=-\frac{12}{5}\left[(2 \mu+3)\left(x-\frac{1}{3}\right)+(\mu-1)\left(y-\frac{1}{3}\right)\right]+1+\varepsilon_{1}\left(x-\frac{1}{3}, y-\frac{1}{3}\right)
$$

and

$$
\beta(x, y)=-\frac{12}{5}\left[(\mu+3)\left(x-\frac{1}{3}\right)+(2 \mu+1)\left(y-\frac{1}{3}\right)\right]+1+\varepsilon_{2}\left(x-\frac{1}{3}, y-\frac{1}{3}\right)
$$

where $\varepsilon_{i}, i=1,2$ is a smooth function such that

$$
\varepsilon_{i}(0,0)=\partial_{x} \varepsilon_{i}(0,0)=\partial_{y} \varepsilon_{i}(0,0)=0
$$

and $\mu$ is a real number. To be consistent with assumption A1 the function $\alpha(x, y)$ and $\beta(x, y)$ have to satisfy $\partial \alpha(x, y) / \partial x \leq 0$ and $\partial \beta(x, y) / \partial y \leq 0$. This implies $\mu \geq-0.5$. It 
is sufficient to restrict the analysis to non negative $\mu$. Then by virtue of Lemma 1 the Jacobian matrix at $m$ is then given as

$$
A(\mu)=\left(\begin{array}{cc}
\mu & -1 \\
1 & \mu
\end{array}\right) .
$$

The solution to the linear differential equation $X^{\prime}=A(\mu) X$ with initial condition $X(0)=(x(0), y(0))$ is given by

$$
\begin{aligned}
& x(t)=\mathrm{e}^{\mu t}(\cos (t) x(0)-\sin (t) y(0)), \\
& y(t)=\mathrm{e}^{\mu t}(\sin (t) x(0)+\cos (t) y(0)) .
\end{aligned}
$$

For $\mu=0$ these are periodic solutions. Thus one may expect a periodic behavior for $x_{t}$, when $\mu$ is close to zero. Indeed

Proposition 5. For $\mu>0$ small enough, there exists a periodic orbit $t \rightarrow \gamma(t)=\left(\gamma_{1}(t), \gamma_{2}(t)\right)$, solution to the differential equation (5) such that

$$
\lim _{t \rightarrow \infty} \operatorname{dist}\left(x_{t}, \chi(\log (t))\right)=0
$$

with positive probability.

Proof. The dynamical system (4) satisfies the assumptions of the Hopf bifurcation Theorem (see e.g. Guckenheimer and Holmes, 1983, Th. 3.4.2). Therefore there exists a linearly stable periodic orbit $\gamma$ for $0<\mu$ small enough. The proposition then follows from Propositions 7, 5 and Corollary 8.9 in Benaim (1999).

Proposition 5 makes a very important point. It establishes that with three standards in competition the stochastic process of adoption might never converge. It might describe cycles between two equilibria, spending some time (possibly very long period of time) close to an equilibrium (e.g., $\left.\gamma_{1}\right)$ and then moving to the other $\left(e . g ., \gamma_{2}\right)$, spending some time there, and then moving again, and so on. These equilibria need not to be standardization ones. They might be incompatibility equilibria.

The global convergence result of Proposition 3 does not hold once we consider more than two standards. This is an important result because the literature on evolutionary game theory and social learning has focused on convergence results. Yet it is relatively easy to find examples where such results do not hold. These examples are not particularly complex, nor 'pathological'. This means that cyclical behaviors are indeed very likely. Then both the literature on standardization and the literature on evolutionary game theory and social learning are missing an important aspect of the dynamics in equilibrium selection.

\section{Conclusion}

Standardization is often the result of successive individuals' choice. That is, standardization is generally market driven and decentralized. This paper studies in a dynamic model inspired by evolutionary game theory the convergence of market share of non proprietary standards. It generalizes the Auriol and Benaim (2000) convergence result in the 
case of two standards. More importantly it shows that in the case of three standards the decentralized process of adoption can oscillate between two equilibria and describe cycles. In other words the convergence result is not robust to the introduction of more than two standards. This suggests that while focusing on equilibrium selection the literature on standardization and the literature on evolutionary game theory and social learning are overlooking a very important aspect of the problem at hand. That is, in practice it is common to have more than two standards in competition. If laissez-faire leads to oscillation among several equilibria, not only standardization is going to fail, but more than this society will fail to reach a stable state. The social costs of such failure are potentially very high. Indeed growing international trade and technological integration increases the need for standardization and conformity assessment systems. In a worldwide integrated economic system individuals have trouble tracing the origin or assessing the impact of their consumption. Standardization failure and oscillations can lead to major consumers confidence crisis, as the one experienced today in Europe with the mad cow disease. The economic consequences of such confidence crisis are clear. The consumers who cannot trust what they consume, reduce their demand which leads to a recession in the sector. If the crisis should generalize because of repeated failures the impact on growth and welfare would be dramatic. Then as a further extension of this study it would be interesting to explore in detailed examples the determinant of cycles in decentralized standardization. This would help us to understand when to expect convergence, or on the contrary oscillations.

\section{Appendix 1. Micro-foundation of individuals' preferences}

Let's consider without loss of generality the case $n=2$. At date $t$ the problem of an adopter is to allocate her demand between standard 1 with market share $x_{1}^{t}$ and standard 2 with $x_{2}^{t}$. That is, she chooses in the set $Q=\{(q, 1-q): q \in[0,1]\}$ a bundle $\left(q_{1}, q_{2}\right)^{8}$. There is a formal analogy between this problem and a problem of choice under uncertainty with outcome being replaced by market share, and lotteries by $q_{1}, q_{2}$. We assume that the individuals' preference over the set $Q$ are rational in the extended Von NeumanMorgensten sense. That is, for any vector of outcome (i.e., for any market shares $\left.x^{t}=\left(x_{1}^{t}, x_{2}^{t}\right)\right)$, the preferences on $Q$ are complete, transitive, continuous, and satisfy the extended independence axiom. Then the extended expected utility theorem implies that these preferences admit a state-dependent expected utility representation: $(q, 1-q)$ $\left(q^{\prime}, 1-q^{\prime}\right) \Leftrightarrow q u_{1 \delta}\left(x^{t}\right)+(1-q) u_{2 \delta}\left(x^{t}\right) \geq q^{\prime} u_{1 \delta}\left(x_{t}\right)+\left(1-q^{\prime}\right) u_{2 \delta}\left(x_{t}\right)$. Contrary to the case where outcome is money leading to a state-independent expected utility representation, we obtain a different function $u_{k}($.$) in every state k=1,2$. Finally, different individuals have different preferences, and the utility functions vary with $\delta$. We assume that

\footnotetext{
${ }^{8}$ Term $q_{k}$ can be interpreted as a quantity (the fraction in total individual's demand dedicated to standard $k$ ), but it can also be interpreted as a probability $\left(q_{1}, q_{2}\right.$ is then a lottery which gives standard $k$ at quality $x_{k}^{t}$ with probability $q_{k}$ ).
} 
$u_{k \delta}\left(x_{t}\right)=\delta_{k} u_{k}\left(x_{t}\right)$. Individual's preference over the set $Q$ associated to standard 1 and 2 with market share $x^{t}=\left(x_{1}^{t}, x_{2}^{t}\right)$, is represented by the following utility function:

$$
U_{\delta}(q, 1-q)=q \delta_{1} u_{1}\left(x^{t}\right)+(1-q) \delta_{2}\left(u_{2}\right) x^{t} .
$$

We deduce that adopters make a discrete choice between standard 1 and 2 . They choose in $Q$ either $(1,0)$ or $(0,1)$, hence $S_{1}{ }_{-\delta} S_{2} \Leftrightarrow \delta_{1} u_{1}\left(x^{I}\right) \geq \delta_{2} u_{2}\left(x^{t}\right)$.

\section{Appendix 2. Proof of Proposition 2}

The proof is based on the following classical lemma.

Lemma 2. Let $X_{1}, \ldots X_{n}$ denote i.i.d random variables having an exponential distribution. That is

$$
\operatorname{Pr}\left(X_{i} \leq t\right)=\int_{0}^{t} \mathrm{e}^{-u} \mathrm{~d} u=1-\mathrm{e}^{-t}
$$

for $t \geq 0$ and

$$
\operatorname{Pr}\left(X_{i} \leq 0\right)=0 .
$$

Let $S=\sum_{i=1}^{n} X_{i}$. Then the random variable $\delta \in \Delta^{n-1}$ defined by

$$
\delta_{i}=\frac{X_{i}}{S}
$$

is uniformly distributed over $\Delta^{n-1}$.

Now, using this lemma one can write

$$
\begin{gathered}
\operatorname{Pr}\left(\delta_{1} U_{1}(x) \geq \delta_{i} U_{i}(x) \text { for all } i\right)=\operatorname{Pr}\left(X_{i} \leq X_{1} \alpha_{i}^{1}(x) \text { for all } i \neq 1\right) \\
=\int_{0}^{\infty} \Pi_{i=2}^{n} \operatorname{Pr} \operatorname{Pr}\left(X_{i} \leq y \alpha_{i}^{1}(x) \mid X_{1}=y\right) \mathrm{e}^{-y} \mathrm{~d} y \\
=\int_{0}^{\infty} \Pi_{i=2}^{n}\left(1-\mathrm{e}^{-\alpha_{i}^{1}(x) y}\right) \mathrm{e}^{-y} \mathrm{~d} y
\end{gathered}
$$

Since

$$
\Pi_{i=2}^{n}\left(1+a_{i}\right)=1+\sum_{k=1}^{n-1}\left[\sum_{i_{1}<\ldots<i_{k} \in\{2, \ldots, n\}} a_{i_{1}} \ldots a_{i_{k}}\right]
$$

we deduce that

$$
k=\int_{0}^{\infty}\left[\mathrm{e}^{-y}+\sum_{k=2}^{n}(-1)^{k} \sum_{i_{1}<\ldots<i_{k} \in\{2, \ldots, n\}} \exp \left(-\left(1+\delta_{1} U_{i_{i}}^{1}(x)+\ldots+\alpha_{i_{k}}^{1}(x)\right) y\right)\right] \mathrm{d} y .
$$




\section{References}

Ainslie G. (1992) Pico-economics. Cambridge University Press, Cambridge.

Arthur W. (1988) Self-reinforcing mechanisms in economics. In: The economy as an evolving complex system. Addison-Wesley Publishing Company, Redwood city, CA, pp. 9-31.

Arthur W. (1989) Competing technologies, increasing returns, and lock-in by historical events, The Economic J., 99, pp. 116-131.

Auriol E., Benaim M. (2000) Standardization in decentralized economies, American Economic Review, 90 (3), pp. 550-570.

Benaim M. (1999) Dynamics of stochastic approximation algorithms. In: Azema J., Emery M., Ledoux M., Yor M. (Eds.), Le Séminaire de probabilités, Lectures Notes in Mathematics 1709. Springer-Verlag, Berlin, pp. 1-68.

Crawford V. (1995) Adaptive dynamics in coordination games, Econometrica, 63, pp. 103-158.

Dupuis P. (1988) Large deviation analysis of some recursive algorithms with state dependent noise, Annals of Probability, 16 (4), pp. 1509-1536.

Ellison G. (1993) Learning, local interaction, and coordination, Econometrica, 61, pp. 10471071.

Farrel J., Saloner G. (1985) Standardization, compatibility, and innovation, Rand. J. of Econ., 16, pp. 70-83.

Fudenberg D., Kreps D. (1993) Learning mixed equilibria, Games and Economic Behavior, 5, pp. 320-367.

Fudenberg D., Levine D.K. (1998) Theory of learning in games. MIT press, Cambridge, MA.

Guckenheimer J., Holmes P. (1983) Nonlinear oscillations, dynamical systems, and bifurcations of vectors fields, Applied Mathematical Sciences, 42, Springer-Verlag, New York.

Kandori M., Mailath G., Rob R. (1993) Learning, mutation, and long run equilibria in games, Economica, 61, pp. 29-56.

Katz M., Shapiro C. (1985) Network externalities, competition, and compatibility, American Economical Review, 75, pp. 424-440.

Loewenstein G., Prelec D. (1992) Anomaly in intertemporal choice: Evidence and interpretations, Quartely Journal of Economics, 107, pp. 573-590.

Weibull J. (1995) Evolutionary Game Theory. MIT Press, Cambridge. 